\title{
Management of Energy in Wireless Sensor Networks with Energy-Starving Sensors
}

\author{
Jobin Varghese ${ }^{1}, \mathrm{~S}$. Viswanatha Rao ${ }^{2}$ \\ ${ }^{1}$ (M.tech Scholar, Telecommunication Engineering, Mar Baselios College of Engineering and Technology, \\ Trivandrum, India \\ ${ }_{2}^{2}$ (Professor\& Head, ECE department, Mar Baselios College of Engineering and Technology, Trivandrum, India
}

\begin{abstract}
Wireless sensor networks provide wide-scale connectivity to the physical world at a lower cost than that of the wired alternative. The complete exploitation of this technology is prevented by various energy factors, which serves as a major barrier. Nodes are powered by various energy sources such as batteries, super capacitors with a limited lifetime or by harvesting energy from the external environment. This means that this limited energy source should be effectively and judiciously utilized. Efficient management of energy is a vital requirement for the optimal design of a wireless sensor network. Most of the literatures propose various energy management strategies, under the assumption that data acquisition consumes significantly less energy than their transmission. But this assumption does not sound good in a number of practical scenarios, where the power consumption for the sensing activity may be greater than that of radio terminal. So energy management schemes should give criteria for the efficient and effective use of energy in energy-starving sensors. In this paper, we survey various methodologies for the efficient management of energy in Energy-Starving sensors
\end{abstract}

Keywords: Adaptive sensing, Duty cycle, Energy Management, Operating System, Wireless Sensor Networks.

\section{INTRODUCTION}

Present trends in communication and embedded technologies enabled the manufacturing of miniature, inexpensive, low-power, multifunctional devices, called sensor nodes. These nodes are low power devices having one or more sensors, provided with a radio. Such a node uses various sensors like mechanical, biological, chemical, optical etc. to monitor different parameters of the environment. Generalized architecture of wireless sensor node is shown in Fig. 1.Generally sensor node consist of a sensing unit, a processing unit, a communication unit, and a power source or energy source. Sensing section consists of a sensor and Analog to Digital Convertor (ADC), where the analog signals produced by the sensors are converted to digital signals by the ADC. It is then given to the processing unit (comprising the processor and storage). The communication unit acts as the link between the sensor node and the network. The power scavenging source generally derives energy from solar and battery power.

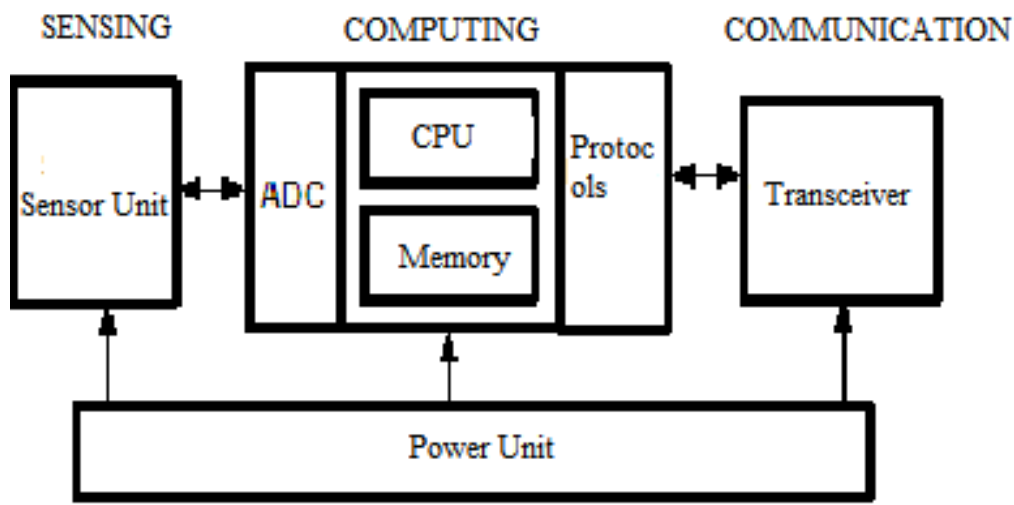

Fig. 1: Generalized Architecture of Wireless Sensor Node

Energy consumption is the most important factor in determining the life of a Wireless Sensor Networks(WSNs), as the sensor nodes are limited by very low energy resources for tasks involving data sensing, processing etc. Therefore optimization of energy techniques in WSNs is essential. This can be addressed by taking into account the issues related to energy awareness in every aspect of design and operation of each sensor node. Different methodologies can be uniquely applied to enhance the life of sensor nodes [1]. For long running operation of WSNs the power from the secondary or primary source should be utilized effectively. Techniques for minimizing the energy consumption in the radio transceiver (transmitting, receiving) might not be sufficient 
to fully address the issues related to energy utilization. Hence energy management schemes, at radio level needs to be complemented with (or replaced by) suitable management schemes for energy-conservation at the sensor level. These techniques operate to reduce data samples rather than the number of transmitted messages.

This Paper gives an overview of design layout of various methods of energy conservation at sensor level. It also gives features of these methods along with a comparison among them. Strategies for reducing the power consumption at the radio level are surveyed in [2] and interested readers can refer to it. Further energy savings can also be obtained by considering data compression [3] [4], predictive monitoring model [5], topology management architecture [6] [7] ,data aggregation [8] [9], adaptive or dynamic duty cycle [10].

The remaining part of the paper is organized as follows. Section II discusses the general approaches to energy conservation at sensor level and introduces the two main approaches (duty-cycling, adaptive sensing). In Section III we break down this high-level classification, highlight the adaptive sensing schemes and their comparison. Finally, the paper concludes with Section IV.

\section{GENERAL APPROACHES FOR SENSOR ENERGY MANAGEMENT}

Sensor networks in applications for monitoring, surveillance rely on a philosophy of synchronous operation, where readings are carried out with a given sampling frequency [11]. Two main approaches can be taken to reduce the energy consumed by a node, i.e., duty cycling and adaptive sensing as shown in Fig 2. In Duty cycling system, sensors wakes up when it needs to acquire a new set of samples and powering it off immediately afterwards. This gives optimal management of energy provided that, the phenomenon to be monitored are timeinvariant and known in advance. Also in duty cycling scheme the sampling rate is larger than necessary, which causes wastage of energy. A more suitable approach is an adaptive sensing strategy, which is capable to dynamically adapt the sensor activity to the real dynamics of the process. The Media Access Control (MAC) scheme has to provide a set of inputs for sleep/awake schedule to support duty cycle mechanisms. Then the application layer monitors the duty cycling scheme so that it can acquire data according to the sensing strategy. This paper gives a detailed description of adaptive sensing techniques.

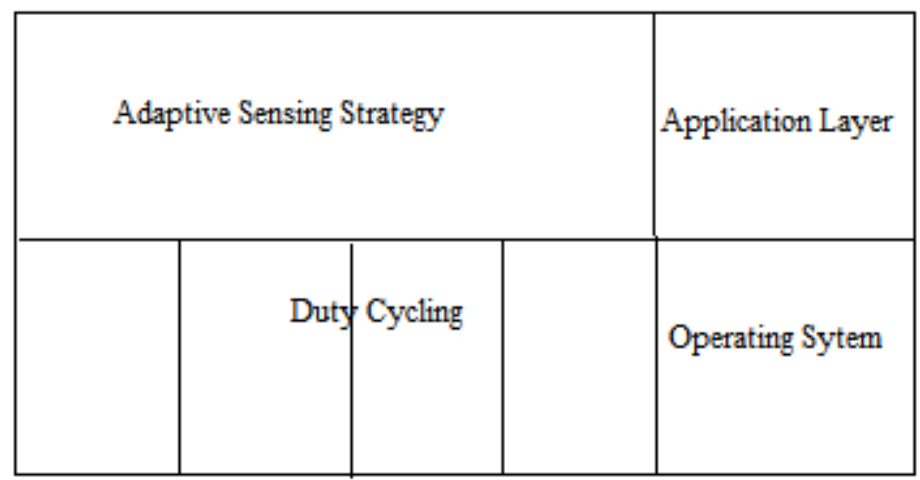

Fig. 2: General Layout of Efficient Energy Management

\section{CLASSIFICATION OF ADAPTIVE SENSING METHODOLOGIES}

The layout for the classification is shown in Fig. 3. Adaptive sensing can be implemented by exploiting three different approaches, i.e., hierarchical sensing, adaptive sampling, and model-based active sensing.

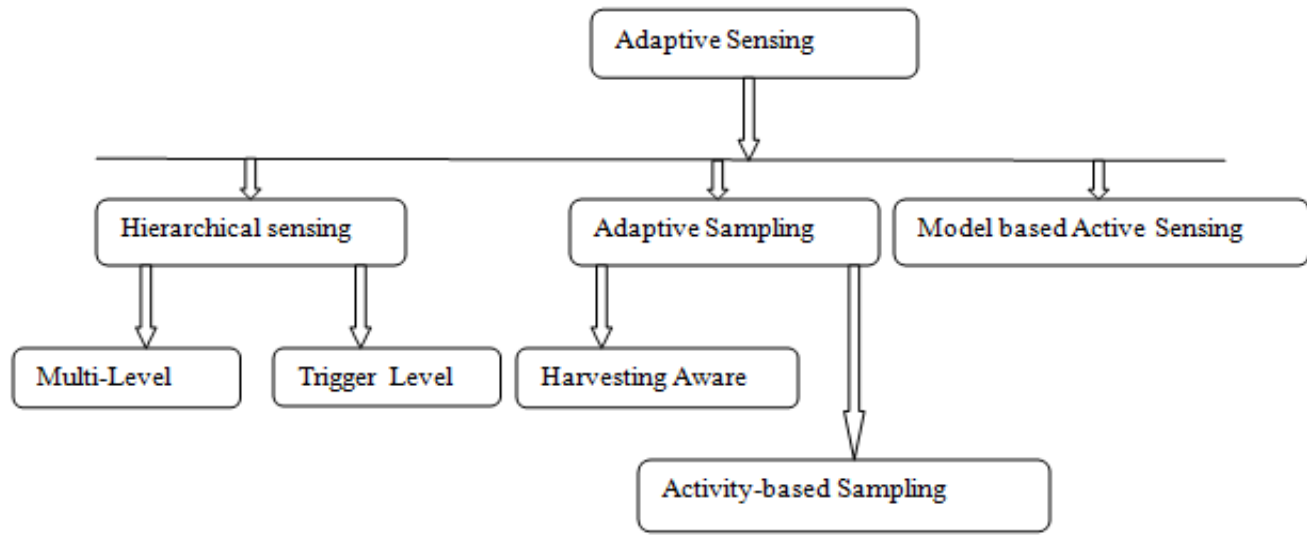

Fig. 3: Classification of Adaptive Sensing Technologies 


\subsection{Hierarchical sensing}

Here different sensors have their own energy, accuracy and power consumption. Final data is obtained by processing the output from different sensor nodes. There is always a conflict between accuracy and energy efficiency. Complex, highly accurate sensors are less energy efficient than the simple sensor nodes. The common setup is that the events are triggered by using the simple sensors and complex sensors are used for measuring the readings. Hence this technique helps to select dynamically the sensors in a sensing field. It can be classified as the trigger level and multi scale/multilevel [12].

\subsubsection{Trigger level}

In this method the complex sensors are actively woken up after detecting an event in the sensing field. The activation of such complex sensors by sensing an event is called trigger level sensing Detection of some activity in the sensing environment is referred to as triggered sensing. Here the structure to be monitored is divided into regions, within which the sensing units are capable of detecting the events. All the activities are controlled by a master node which controls all the activities of the network.

3.1.2 Multi-Level

Hierarchical method can be effectively be utilized if we know the region within the sensing field which requires more accurate observations. This is obtained by depending on the layout of the sensing network having a mixture of simple and complex nodes. Hence highly precise ones are put in areas where accurate data collection is required. Such methods which use the above features are called multi-level.

3.2 Adaptive sampling

Here the sensor sampling rate is dynamically adjusted based on correlation of data based on temporal/spatial domain and amount of residual energy, when the node is able to harvest from the surroundings. 3.2.1 Activity Based sampling

Activity-driven adaptive sampling exploits the correlation (both temporal and spatial) among the acquired data as shown in Fig. 3.Here the sampling rate is adjusted in accordance with the physical environment. A spatial correlated approach has been investigated in [13] where the authors introduce the backcasting scheme. Here, the main view is that nodes are not necessarily to sense the field in a uniform way. The idea behind is that more nodes should be active in those areas where the statistical variation of the reading is high In this scenario the process of waking up the necessary number of sensors is done in two steps. In the first step only a subset of nodes are activated and statistical estimate of sensing is evaluated. Several steps are involved in the estimation process. At first, the sensors are activated within a sensing field in a number of subsquares with a non-uniform resolution; the smaller the spatial variation of the observed phenomenon the larger the sub-square associated with the location. Then clusters are formulated, each of which is controlled by a cluster-head. Finally, the data is sent to a fusion center. The second step is called refinement; the fusion center may activate additional sensors in the areas where the spatial correlation among the data is low. This is realized by a "backcast" procedure where the fusion center sends an activation message to those cluster-heads residing in the smallest square areas generated in the first phase. In this procedure more nodes are active in those areas where the variation of the sensing parameter is high. A spatial Correlation-based Collaborative MAC protocol (CC-MAC) is suggested in [14] which controls medium access and prevents redundant transmissions from closely located sensors. The information obtained by the Iterative Node Selection is broadcast to sensor nodes during the network setup and it is used during the operational phase. CC-MAC also prevents of redundant information transmission.

Temporal correlation approach has been investigated in [15], where the authors proposed an adaptive sampling algorithm for minimizing the energy consumption of a sensor in a snowy environment. The suggested algorithm dynamically estimates the current maximum frequency of the signal.

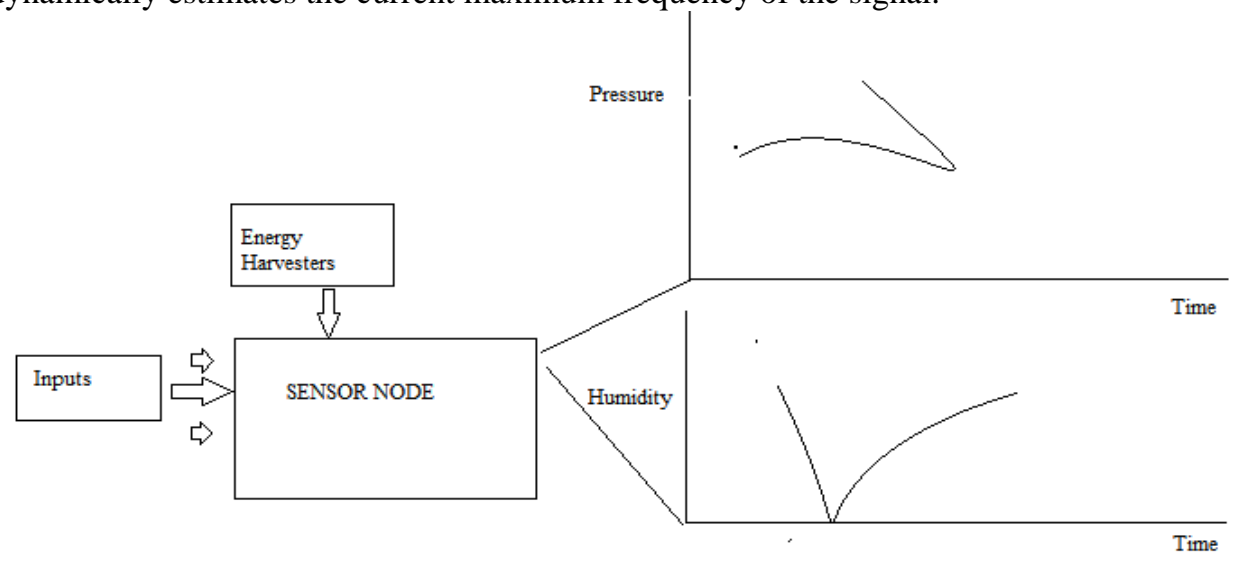

Fig. 3: Activity based Sampling 


\subsubsection{Harvesting-aware sampling}

The harvesting-aware adaptive sampling method [16] takes into account the residual and the forecast energy coming from the harvester system. The approach requires the development of models to characterize the increase in energy with time and the energy consumption of sensor units. The non ideality of the system of harvesters has been modeled by considering both the loss in charging operation due to the non-ideal charging efficiency and the leakage current of the energy storage. The problem related to energy variation has been reformulated as a tracking of linear-quadratic decision which is solved with a simple ad-hoc control law [17].

\subsection{Model-based Active Sensing}

In this method,the proposed model predicts next, hence avoids sampling at the nodes in regular intervals. Both nodes and sink can be modeled to be of the same characteristics. The optimality of this approach is obtained from the accuracy of the model and the nature of the process to be monitored. The advantage of this type of modeling is that model-based active sensing reduces the energy needed for the acquisition of data and also decreases the amount of data that is sent to the sink by discarding the redundant data.

This type of approach was first proposed in [18] in the framework of the Barbie-Q (BBQ) query system. Here the approach is such a way that the query system has a probabilistic model and a query planner, both present in the sink. A probability density function (pdf) over a set of attributes is derived, spatial and/or temporal correlations are exploited from these values. Query plan and other relevant data are built by planner. For example, when a user wants to evaluate the temperature, the planner selects the subset of sensors to be contacted and the quantities to be sampled.

Another approach has been suggested in [19], where an Adaptive Sampling Approach to Data Collection (ASAP) is introduced. In contrast with BBQ, ASAP splits the network into Group of sensors called clusters. Cluster heads are elected by cluster formation head. Statistical correlation of sensor readings and the hop count determines the factors for forming the cluster. The correlation-based sampler selection is performed at each cluster head and aims to determine the sampler nodes that capture the best spatial and temporal correlations among the other sensor readings. Sensor readings are collected from sampler nodes that have been selected.

Besides the sensing model, the author also defines a routing protocol which takes into account the energy spent for both sensing and communication. Additional sampling is performed in Sensors that are not relaying. The routes where data is sampled with lower frequency are the routes where nodes spend more energy for sampling.

Table 1 shows the comparison of the above described energy management schemes. Here the sensors are identified based on complexity.

Table 1: Comparison of Energy Efficient Mechanisms at Sensor Level.

\begin{tabular}{|c|c|c|c|c|c|}
\hline Category & Methodology & Type of Sensors & $\begin{array}{c}\text { Data } \\
\text { accuracy }\end{array}$ & $\begin{array}{c}\text { Energy } \\
\text { Efficiency }\end{array}$ & Applications \\
\hline $\begin{array}{l}\text { Hierarchical } \\
\text { Sensing. }\end{array}$ & $\begin{array}{l}\text { Activation takes on } \\
\text { the detection of } \\
\text { event. }\end{array}$ & $\begin{array}{l}\text { Simple and } \\
\text { complex sensors } \\
\text { are used within } \\
\text { the same } \\
\text { network. }\end{array}$ & High. & Moderate & $\begin{array}{l}\text { Object/event } \\
\text { detection, } \\
\text { environmental } \\
\text { monitoring. }\end{array}$ \\
\hline Adaptive Sampling & $\begin{array}{l}\text { Sampling rate is } \\
\text { changed by } \\
\text { calculating the } \\
\text { correlation at } \\
\text { spatial/temporal } \\
\text { domain. }\end{array}$ & $\begin{array}{l}\text { Same kind of } \\
\text { sensors. }\end{array}$ & Moderate. & High. & $\begin{array}{l}\text { Energy } \\
\text { Harvesting- } \\
\text { WSNs. }\end{array}$ \\
\hline $\begin{array}{l}\text { Model based Active } \\
\text { Sampling }\end{array}$ & $\begin{array}{l}\text { Predictive model to } \\
\text { predict next data. }\end{array}$ & $\begin{array}{l}\text { Same kind of } \\
\text { sensors. }\end{array}$ & Moderate. & Moderate. & $\begin{array}{l}\text { Specific } \\
\text { Scenario. }\end{array}$ \\
\hline
\end{tabular}

\section{CONCLUSION}

In this paper various approaches for energy conservation in wireless sensor networks at sensor level has been investigated. We think that the field of energy conservation in data acquisition and processing has not been fully explored yet, so that there is room for developing convenient techniques to reduce the energy consumption of the sensors.. Another interesting point is that most of the solutions proposed in this paper 
assumes that the energy consumption of the sensors is much higher than the energy consumption at radio terminal. This assumption matches with the many of the real world applications. Data accuracy, energy efficiency and applications of various categories of energy management schemes are illustrated in Table 1.

\section{REFERENCES}

[1] G. Anastasi, M. Conti, M. Di Francesco, A. Passarella, "How to Prolong the Lifetime of Wireless Sensor Networks", Chapter 6 in Mobile Ad Hoc and Pervasive Communications, (M. Denko and L. Yang, Editors), American Scientific Publishers, to appear, available at http://info.iet.unipi.it/ anastasi/ papers/Yang.pdf.

[2] G. Anastasi, M. Conti, M. Di Francesco, A. Passarella, "Energy Conservation in Wireless Sensor Networks", Ad Hoc Networks, to appear (currently available at http://info.iet.unipi.it/ anastasi/papers/adhoc08.pdf).

[3] C. Tang, C. Raghavendra, "Compression Techniques for Wireless Sensor Networks", Chapter 10 in Wireless Sensor Networks, Kluwer Academic Publishers, Boston, USA, 2004.

[4] C. Sadler, M. Martonosi, "Data Compression Algorithms for Enegy-Constrained Devices in Delay Tolerant Networks", Proc. ACM SenSys 2006, Boulder, Colorado, USA, November 2006.

[5] S. Goel, T. Imielinsky, "Prediction-Based Monitoring in Sensor Networks: Taking Lessons from MPEG", ACM Computer Communication Review 2001, Vol. 31, N. 5, October 2001.

[6] A. Cerpa, D. Estrin, “ASCENT: Adaptive Self-Configuring Sensor Networks Topologies”, Proc. IEEE Infocom 2002, New York, USA, 2002.

[7] C. Schurgers, V. Tsiatsis, S. Ganeriwal, M. Srivastava, "Optimizing Sensor Networks in the Energy-Latency Density Design Space", IEEE Trans. on Mobile Computing, Vol.1, N.1, 2002.

[8] S. Madden, M. Franklin, J.Hellerstein, W. Hong. "TAG: a Tiny AGgregation Service for Ad-Hoc Sensor Networks", Proceedings of OSDI, 2002.

[9] A. Boulis, S.Ganeriwal, M. B. Srivastava, "Aggregation in Sensor Networks : an Energy-Accuracy Trade-off”, Ad Hoc Networks, Vol. 1 (2003), pp. 317-331.

[10] D. Ganesan, A. Cerpa, W. Ye, Y. Yu, J. Zhao, D. Estrin,, "Networking Issues in Wireless Sensor Networks", Journal of Parallel and Distributed Computing, Vol. 64 (2004).

[11] Romer, K.; Mattern, F., "The design space of wireless sensor networks," Wireless Communications, IEEE, vol.11, no.6, pp. 54-61, Dec. 2004

[12] Cesare Alippi, Giuseppe Anastasi, Mario Di Francesco,, Manuel Roveri” Energy Management in Wireless Sensor Networks with Energy-hungry Sensors", IEEE Instrumentation and Measurement Magazine 'Vol. 12, N. 2, April 2009, pp. 16-23

[13] R. Willett, A. Martin, R. Nowak, "Backcasting: Adaptive Sampling for Sensor Networks", Proc. International Symposium on Information Processing in Sensor Networks (IPSN 2004), pp. 124-133, 26-27 April 2004.

[14] M. C. Vuran and I. F. Akyildiz, "Spatial Correlation-based Collaborative Medium Access Control in Wireless Sensor Networks", IEEE/ACM Transactions on Networking, Vol. 14, No. 2, pp. 316-329, April 2006.

[15] C. Alippi, G. Anastasi, C. Galperti, F. Mancini, M. Roveri, "Adaptive Sampling for Energy Conservation in Wireless Sensor Networks for Snow Monitoring Applications”, Proc. IEEE MASS 2007, Pisa, Italy, October 8, 2007.

[16] A. Kansal, J. Hsu, S. Zahedi, M. Srivastava, "Power Management in Energy Harvesting Sensor Networks, ACM Transactions on Embedded Computing Systems, Vol. 6, N. 4, 2007.

[17] C. Vigorito, D. Ganesan, A. Barto, "Adaptive Control of Duty-Cycling in Energy-Harvesting Wireless Sensor Networks, Proc. IEEE Conference on Sensor, Mesh, and Ad Hoc Communications and Networks (SECON 2007), San Diego, CA, 2007.

[18] A. Deshpande, C. Guestrin, S. Madden, J. M. Hellerstein, W. Hong, "Model-Driven Data Acquisition in Sensor Networks", Proc. International Conference on Very Large Data Bases (VLDB 2004), Toronto, Canada, 29 August - 3 September, 2004.

[19] B. Gedik, L. Liu, P. S. Yu, "ASAP: An Adaptive Sampling Approach to Data Collection in Sensor Networks", IEEE Trans. Parallel Distributed Systems, Vol. 18, N. 12, December 2007. 